\title{
A Systematic Review of Way That Human Body Can Be an Interface of Information Exchange
}

\author{
Jingyun Huang ${ }^{1, *}$ \\ ${ }^{1}$ Wuxi Foreign Language School, Wuxi, Jiangsu Province, China \\ ${ }^{*}$ Corresponding author. Email: guanghua.ren@gecacademy.cn
}

\begin{abstract}
Mediums of information transfer around the world are diverse. However, as the simplest interface, human bodies are usually ignored. The human body can easily deliver various information to outside. Here, the purpose of this paper is to investigate how the significant factors of technology and visual culture enable the human body to express relevant information about them. This article will do literature review, analyze some experiments and a lot of media records like photos experiment data, and movies. Advanced technologies are also introduced and used in this paper.

This article reveals that human bodies can be an interface of information exchange. First, humans' nervous system can be analyzed by the latest equipment which can detect brain activities. These records offer content about people's thoughts and ideas, which means the nervous system of a human reflects the information. In the next part, this paper will concentrate on cell phones. A lot of pictures and photos on the Internet in cell phones are able to show different information like jobs and identity. Finally, diverse types of scenarios, lens, and environments in VR glasses and science fiction involved in horrible scenes can easily change people's behaviors and responses. Thus, the result consists of the evidence that is used.

The paper is helpful to people who want to research different functions and connections between the human body and visual culture.
\end{abstract}

Keywords: Human body, Visual culture, Nervous system, Visual reality, Science fiction.

\section{INTRODUCTION}

Is the human body a simple object? People use their bodies to watch, listen, eat, smell and exercise every day. But most of us don't seem to notice that our bodies can transfer all kinds of information to the outside world. Researchers have been focusing on analyzing and inferring human psychology or information they want to convey through their behavior and expressions. Therefore, some forms of visual culture, which make human bodies an interface of information exchange, are able to bring people the most intuitive and direct feeling. The earliest article that mentioned "visual culture" is " The Art of Decorative Painting"[1]. At that time, visual culture meant architecture and painting. Later the word "visual culture" began to change and develop different forms. As Jinjin Liu maintains that Visual culture developed from single art to some non-artistic objects later [2]. In modern society, the concept of visual culture is the way that individuals view the world through a visual text. Visual text includes various forms. For instance, movies, photos, and paintings. In Ming Fu's paper, she claims that visualizing what is not visual is one of the notable features of visual culture[3].

Now the technology is getting more mature and all kinds of high-tech methods are applied to things like photography, advertisement, and movies. These technologies belong to forms of visual culture because people are able to find a lot of information including world views throughout them. Like Ming Fu claims that with the development of modern science and technology, the industrial revolution, the invention of photography and lithography have led to the mass production of goods and images, as well as the arrival of the digital era predicted by computers. It also indicates that richer materials will become the latest media and theme of today's artists [3]. The computer is a typical technology. It can provide a large number of forms of visual text involved in movies, videos, and E-books. It connects our tradition and new world. From the same article of Ming Fu, she believes that computer enables artists to obtain new ways of expression. It creates the 
reconstruction of design and provides a great space and rich forms for visual culture [3]. Technology provides an opportunity for all kinds of new artistic forms to develop and improve. Moreover, participation of people, children or the old is getting much higher gradually. So it is easier for people to change their behaviors including the nervous system and make a response. At this time, it is better to reflect the human body as the interface of information exchange.

Human bodies contain a lot of functions in the world of visual culture. However, there are not many articles that investigate human bodies as an interface of information exchange. Therefore, this paper will try to find some useful information and researches to explore this topic.

The purpose of the paper is to find how visual culture enables human bodies to become the interface of information by summarizing and analyzing some journals and articles. All of the paper is divided into two big parts. They are technology and the nervous system and VR and science fiction.

\section{TECHNOLOGY ALTER THE BEHAVIOR AND NERVOUS SYSTEM OF PEOPLE}

\subsection{Brain wave and human-beings' Nervous System}

Technology improvements are the easiest and direct measure to transfer not only our daily life but also human body. Like Pang said that he found that photography may introduce a new measure of looking at gender, that is, to convey meanings by mediation of technology, as well as the surrounding social and culture ideology [4]. Although he just said way of seeing gender, a lot of people believe technology can alter human bodies including the nervous system.

Here, the experiment about the brain wave on advertisements made by Nielsen, a world-renowned market research company, and Colombia Broadcasting System ( CBS ) will be analyzed.

The motivations and thoughts of human behaviors are complex and sometimes curious. Whether people can be attracted by something like products or services cannot be detected on people's faces only. Some activities in the human's brain are needed to be explored. This is because the brain contains a lot of functions that are related to people's consuming behavior. For instance, Hippocampus, marginal lobe and anterior cingulate gyrus are parts that are responsible for remembering. So if some advertisements are creative and have memory points, audiences may notice and be attracted by the advertisement. Some other regions of the brain are related to the preference of products, evaluation of products, willingness to pay, feeling of experience and decision-making level. Nowadays, lots of researchers have begun to investigate neuroscience. Like Lei Wang and other people point out in their research paper that consumer neuroscience gradually spans from the research on the correlation between cognitive activities and decision-making behaviors to the use of cognitive indicators that in order to predict the upcoming decision-making behaviors of consumers [5]. All kinds of advanced types of equipment are able to reach the goal that studying human brains. In order to attract consumers' attention and to grasp their hearts, Nielsen company begins to research consumer behaviors from the perspective of neuroscience because mood and feelings are the significant factors that can control whether people purchase the products. As in figure 1, researchers capture the feelings and responses of audiences by measuring Electroencephalogram ( EEG ), Facial Coding, Eye tracking, heart rate, etc. By doing this, researchers can learn whether people like this advertisement and whether people are attracted by the brand.

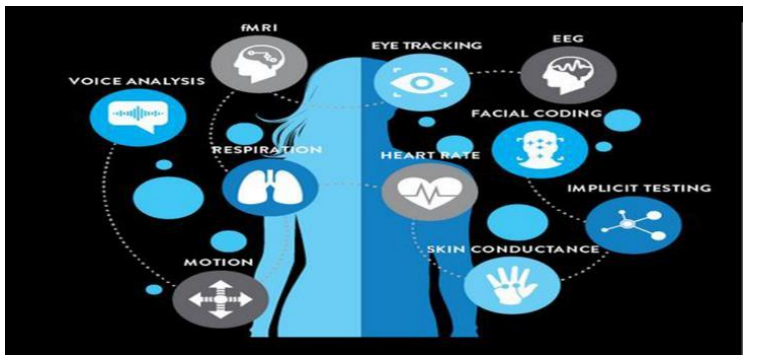

Figure 1 Methods of measuring consumers' nervous system [6]

Here is a particular case of the advertisement of $M$ \&Ms. Researchers measured the Engagement, Electroencephalogram and Facial Coding of consumers. During different parts, the Engagement and Electroencephalogram curve altered. For example, in figure 2 Audiences' Engagement \& Electroencephalogram of plot 1, the curve began to rise when people see the plot that the husband heard his wife was communicating with another voice. Meanwhile, the code of facial expression also changed relatively drastically.

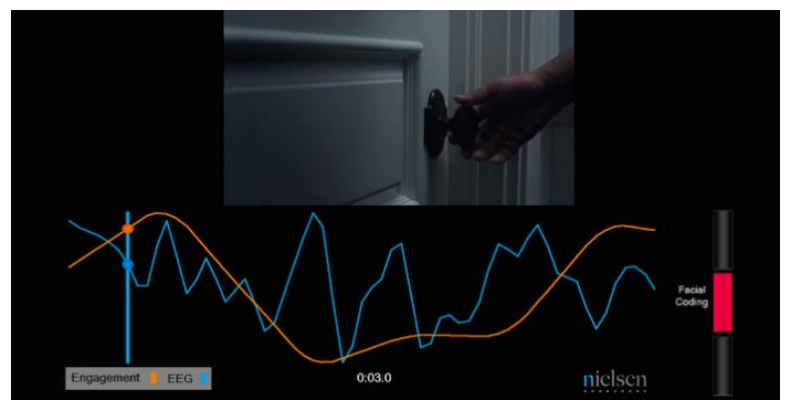

Figure2 Audiences' Engagement \& Electroencephalogram of plot 1 [6] 
As figure 3 Audiences' Engagement \& Electroencephalogram of plot 2shown above, when the husband opened the door and the wife got up from the bed in a hurry, the curves of Engagement and Electroencephalogram reached a peak, which reflects that this part draws people's attention and make them interested in this advertisement.

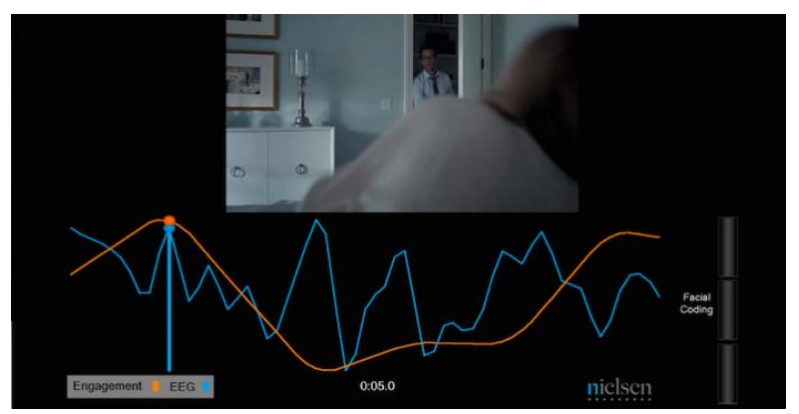

Figure 3 Audiences' Engagement \& Electroencephalogram of plot 2[6]

Another higher peak appeared when the wife began to explain something to the husband in figure 4 Audiences' Engagement \& Electroencephalogram of plot 3. The explanation is rather pale and ambiguous. It's hard for audiences not to think that wife has done some inappropriate things. This situation shows that this kind of plot draws the attention of most people.

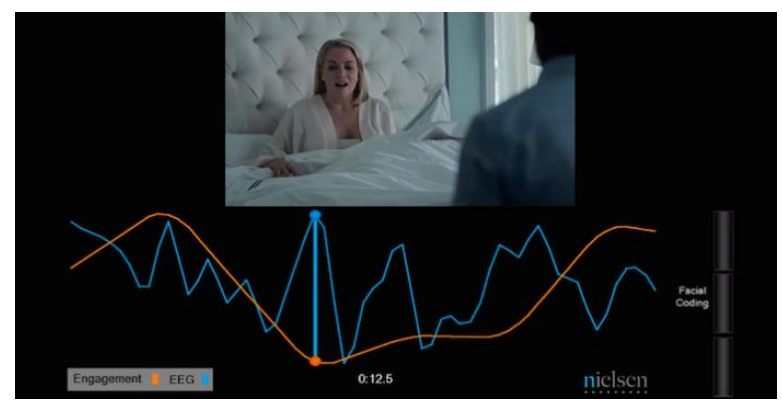

Figure4 Audiences' Engagement \& Electroencephalogram of plot 3[6]

At last, the audience didn't react violently to the appearance of that yellow M \& Ms like figure 5 Audiences' Engagement \& Electroencephalogram of plot 4 showed.

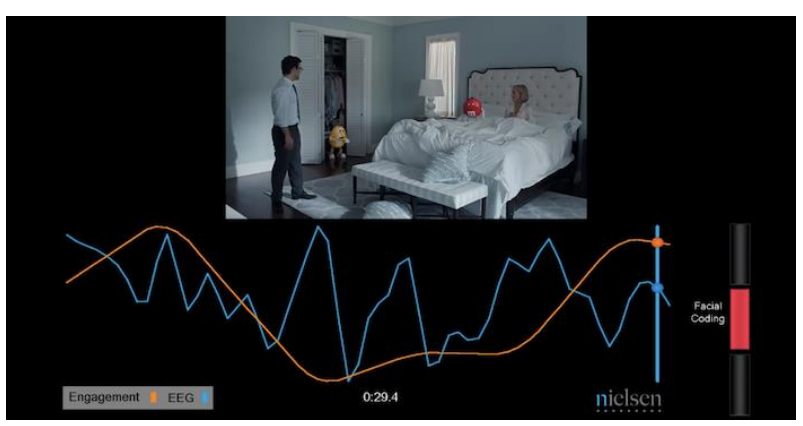

Figure 5 Audiences' Engagement \& Electroencephalogram of plot 4 [6]
Then, according to the data, the company may decide to eliminate the parts that people do not like. Thus, this experiment shows that human bodies especially nervous systems change with the plot of advertisements then researchers record those changes and find out the less popular part. It is clear that the human body, as an interface of information exchange, can provide various information to marketing companies.

\subsection{Cell Phones and human-beings' behaviors.}

Technology influences human life greatly and people pay more attention to spiral entertainment and satisfaction. Cell phones become necessities around us. At the same time, we can gain and learn massive information from them. This information attracts people all the time. In other words, human bodies can be transferred by cell phones because most superficially, some people become addicted to cell phones and they focus on the screen for a long time. This is also a change. However, cell phones can make human bodies the information interface as well. During the paper of Zhenbo Meng, he said that in the platform of mobile phone vision, the identities of communicator and audience can be exchanged, the audience is not passively accepted, and information communicator is not mechanically transmitted. In the communication between the two sides, their roles are constantly changing, and a person can be both communicator and audience. The unprecedented strong interactivity of mobile phone visual communication platforms makes information spread more and more widely [7]. Because of that, from the information from cell phones, for instance, messages from friends or family, people can imagine opposite sides' moods and feelings. They may know what their friends think through their facial expressions without communicating face to face.

Besides, people usually judge the personalities, career and other information of one in their ideas by his or her clothes and facial appearance in photos and videos. Some people may be thought evil by others but evil people can also be thought friendly through the smile they pretend. This confirmed the sentence that Paglen in his article, he claimed that people can create so-called inferior people by using those colonial pseudosciences such as phrenology and physiognomy [8]. From the book "Body And Archive", police will establish an archive for criminals including photos from all aspects people can observe their height, front face and side face[9].

On the other hand, photography is also a significant function of cell phones. People record beautiful views and scenes around them by using phones to take photos. In Bing Gao's article, the author said that communication of image language is also a kind of lifestyle in this modern society [10]. 
Meanwhile, by observing the environment, colors and characters in a photo, we can read the feelings and individualized thoughts of the author. This is also belongs to information that the authors of photos show to others.

Therefore, a person in a photo or the person who takes photos can easily leave information and characteristics about them and people can easily see those photos and videos on their cell phones.

\section{TECHNOLOGY ALTERS THE RESPONSES OF PEOPLE}

\subsection{VR and science fictions and human-beings response}

For a long time, humans pursue bringing virtual objects to human beings to see and watch and they did. An advanced technology called VR enters our life. Virtual reality is a three-dimensional virtual world created by computer simulation, which provides users with the simulation of senses such as sight, hearing and touch so that users can observe things in three-dimensional space in time without restriction [11]. In the real world, people will be fear, scream and hide when they meet something they are afraid. Nevertheless, facing those virtual and nonexistent things, human bodies will make a respond. For example, many games in VR provide a situation that players need to defeat mutated humans. The moment that players see those horrible and different people in the game, VR makes these images look real. At this time, people may be afraid and go back, find something that can support their shivering bodies, or take off the VR glasses. These actions are all the information that shows us that they fear those virtual images. As Nan Fang demonstrates that in the process of watching videos, we reconstructed an in-vivo information exchange and man-machine communication that can be carried out in the virtual image world [12].

Science fictions are the most representative movies that can reflect technology development. Modern movie-making technology can synthesize many fantastic shots. All kinds of advanced special effects are attractive. Thus, people definitely show their own feelings and transmit information about their ideas of the movie. They feel joyful and sometimes show smiles as they see the things they like and be interested in; they are afraid also as they see some bloody and violent scenes.

Take the movie "I Am Legend" as an example. Zombies in the movie like figure 6 do not exist in the real world. In that movie, the production group uses technology to make those images.

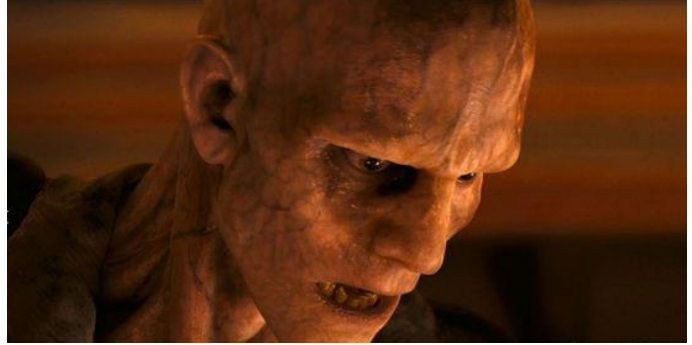

Figure 6. Zombies in I Am Legend [13]

When some of the people watched the movie and saw the zombies for the first time, they just covered their eyes and dared to look. So that is information that reflects their fear. Or the example of "Spider Man". The gene of spider man is altered by that strange spider. There are small thorns on his fingers, which can help him climb on walls. He can jump high and run fast. He also can produce sticky spider silk. People will praise and admire spider man for his just deeds. Actually, 3D technology is a similar principle as VR technology and three-dimension glasses play the same role as VR glasses. Although their operating principles are different, the effects are similar. Three dimension glasses are also able to create an environment that is similar to the real world. Hence, human bodies are altered by technology and people's feelings are controlled by those movies.

\subsection{Observation by others and human-beings' behaviors}

Not only physical contact can transfer human bodies, but also visual impact and unpredictable events. For example, if people think of being monitored by cameras or being supervised by people, they will feel uncomfortable and then change their behaviors. Another famous experiment is Hawthorne Effect. Hawthorne experiment is a famous Management Psychology experiment. The original purpose of an experiment is to research the relationship between work environment including illumination intensity, humidity, rest time, working pressure, working time and the leadership of manager and productivity in Hawthorn factory, which already has had relatively perfect recreation equipment, health care and pension but still low productivity in Chicago. However, when those employees knew that they were observed by researchers, they worked harder than usual because they wanted to show their better working conditions to prove that they are excellent enough so that the productivity was higher. Another example is about children. In a school, every child accepted an intelligence test. Nevertheless, the school made a mistake. Normal students were put in a better class but talented students were put in a normal class. Surprisingly, the scores of those normal students were as outstanding as past talented students. Later, researchers found that these normal students believed 
that they were excellent and notable because teachers pay a lot of attention to them. These two cases show people who realize that they are being observed by others have a tendency to change their behavior, which means people change their behavior this time due to the thoughts that they want to show their better side to others.

\section{CONCLUSION}

This overall paper focuses on investigating the association between human bodies and visual culture. The world of visual culture is colorful and varied. Not only some artworks, but also all kinds of novel expression forms like photos, videos, movies, and advertisements, can inspire people to show their feelings and thoughts through their bodies. Technology is a crucial and indispensable factor to analyze the topic. Technologies nowadays are advanced and strong. Such technologies that are related to visual culture especially VR technology and equipment that can measure the nervous system of people are able to bring people different and new experiences for sensory organs, so as to stimulate people to deliver information unconsciously.

This article explores how human bodies can be an interface of information exchange, especially in visual culture by summarizing some papers and analyzing some examples: First, the paper analyzes the example of Nielsen, a world-renowned market research company, and Columbia Broadcasting System about the brain wave experiment on advertisements. This experiment indicates that the nervous system of humans can reflect some information and it can also be altered. This is because, during different plots in an advertisement, the Engagement and Electroencephalogram curve demonstrate different patterns. As a result, people find what kind of plot do audience like most or least. Then advertisement makers can make appropriate adjustments. Then, this article proves that human behaviors are related to cell phones. The most common change in behaviors is a long-term focus on screens of cell phones. In addition, details in photos and videos about people provide others with information about characteristics and occupations. Finally, by analyzing VR technology and some science fiction, the article claims that when the human face or see virtual objects through VR or three-dimension glasses, some responses of them can express their ideas and feelings. For instance, scream, shiver, and hide. These responses are the signals of fear.

Human bodies are related to all walks of life. By researching diverse functions of human bodies, some people might obtain some useful measures that enable them to achieve their purposes and goals. For instance, some sellers may realize how to catch consumers' attention and some designers and producers can create products that people are interested in. Maybe this paper can provide some enlightenment about the relationship between human bodies, technology, and visual culture to people who meet difficulties on the way to reaching their goals.

\section{REFERENCES}

[1] Walter Bayes. (1927) The Art of Decorate Painting. London: Chapman and Hall Ltd.

[2] Jinjin Liu. (2011) The Name of Visual Culture. Art Research,000(001), 102-108.

[3] Ming Fu. (2008) The New Trend of Visual Culture Under The Development of Modern Society And Technology. Science Association Forum, 000(002), $145-145$

[4] Goldstein, J. . (2009). The distorting mirror: visual modernity in china. American Anthropologist, 111(4), p.518-519.

[5]Lei Wang, Yikai Yang, Jiehui Zheng, Xiaoyi Wang. (2020). Predicting Consumer Behavior From The Perspective of Consumer Neuroscience: Current Situation, Challenges And Future. Journal of Management Engineering, v.34;No.135(06), 6-17.

[6] Qiu Yue Yang. (2016). In order to catch your eye, advertisers have begun to study brain marketing.[Online]

https://www.jiemian.com/article/711299.html.com

[7] Zhenbo Meng. (2015). Visual communication analysis of mobile media. News Research Guide, 000(006), 203-203.

[8] Paglen, T., \& Downey, A. . (2020). Algorithmic anxieties: trevor paglen in conversation with anthony downey. Digital War, 1(1-3).

[9] Allan Sekula (1986) The Body and the Archive. The MIT Press.

[10] Bing Gao (2017) On Mobile Phone Photography and Photography Art in the Visual Age. Art Book,(6).

[11] China Science Communication. [Online] https://baike.baidu.com

[12] Nan Fang. (2016). Visual experience and cultural metaphor of "immersive communication" of VR video. Media 000(010), 75-77.

[13] Baidu. [Online] https://image.baidu.com 